\title{
Local and asymptotic regularity results for quasiconvex and quasimonotone problems
}

\author{
Menita Carozza* Antonia Passarelli di Napoli ${ }^{\dagger}$ \\ Thomas Schmidt ${ }^{\ddagger} \quad$ Anna Verde ${ }^{\S}$
}

October 19, 2010

\begin{abstract}
Considering vectorial integrals in the multidimensional calculus of variations and quasilinear elliptic systems of PDEs, we prove gradient regularity of minimizers and weak solutions, respectively. In contrast to the classical theory we impose our assumptions on the structure functions only locally (i. e. near a single point) or asymptotically (i. e. near infinity). In particular, we point out relations between the local and the asymptotic point of view, and we discuss notions of quasiconvexity at infinity and quasimonotonicity at infinity, which arise in this context.
\end{abstract}

\section{Introduction}

We study multidimensional variational integrals

$$
F[u]:=\int_{\Omega} f(D u) d x \quad \text { for } u: \mathbb{R}^{n} \supset \Omega \rightarrow \mathbb{R}^{N},
$$

where the dimensions $n \geq 2, N \geq 1$, and an open bounded subset $\Omega$ of $\mathbb{R}^{n}$ are fixed for the remainder of the paper. The integrand $f: \mathbb{R}^{N \times n} \rightarrow \mathbb{R}$ is subject to a set of hypotheses that will be imposed below. Actually, minimal assumptions are that $f$ is Borel measurable and satisfies the growth condition

$$
|f(z)| \leq L(1+|z|)^{p} \quad \text { for } z \in \mathbb{R}^{N \times n}
$$

for some exponent $p$ and a constant $L$. Here, the growth exponent $p$ will also be fixed for the remainder of the paper and will always be assumed to satisfy $\mathbf{1}<\boldsymbol{p}<\infty$. In this setting $F[u]$ is well-defined and finite for every function $u$ in the Sobolev space $W^{1, p}\left(\Omega, \mathbb{R}^{N}\right)$. Thus, we may give the following definition of a minimizer of the Dirichlet problem:

Definition 1.1 (Minimizer). Suppose that $f$ is Borel measurable with growth (1.2). We say that $u \in W^{1, p}\left(\Omega, \mathbb{R}^{N}\right)$ is a minimizer of $F$ iff $F[u] \leq F[u+\varphi]$ holds for all $\varphi \in W_{0}^{1, p}\left(\Omega, \mathbb{R}^{N}\right)$.

\footnotetext{
* M. Carozza: Dipartimento Pe.Me.Is, Università degli studi del Sannio, Piazza Arechi II, 82100 Benevento, Italy, e-mail: carozza@unisannio.it

${ }^{\dagger}$ A. Passarelli di Napoli: Dipartimento di Matematica e Applicazioni "R. Caccioppoli", Università di Napoli "Federico II", Via Cintia, 80126 Napoli, Italy, e-mail: antpassa@unina.it

$\ddagger_{T}$. Schmidt: Department Mathematik, Friedrich-Alexander-Universität Erlangen-Nürnberg, Bismarckstr. 1 $\frac{1}{2}$, 91054 Erlangen, Germany, e-mail: schmidt@mi.uni-erlangen.de

$\S$ A. Verde: Dipartimento di Matematica e Applicazioni "R. Caccioppoli", Università di Napoli "Federico II", Via Cintia, 80126 Napoli, Italy, e-mail: anverde@unina.it
} 
It is well-known that key conditions to be imposed on the integrand $f$ are versions of strict convexity. Actually, in this paper we will mostly be concerned with the notion of strict quasiconvexity, a generalization of strict convexity, which has nowadays become a common condition in the vectorial calculus of variations. We mention that quasiconvexity was originally introduced by Morrey [43] and postpone the precise definition to Section 2.1. The basic existence and partial regularity results for minimizers have first been established for strictly convex integrands $[44,32,39]$ and have later been extended to strictly quasiconvex integrands $[43,1,19,27,29,2,28,8,6,15,7,38,16,40,22]$. Here, partial regularity of a minimizer $u$ means that $u$ is smooth outside a negligible set. We stress that in the general vectorial case — which we are treating here - the possible occurrence of singularities has been demonstrated in a series of striking counterexamples (see for instance $[13,46,52,45])$. Thus, partial regularity can in general not be improved to full regularity on the whole domain $\Omega$.

A natural question which may be asked at this stage and which is in the focus of this paper is the following:

Whether - or to what extent - regularity breaks down if the integrand is strictly (quasi)convex only near some points in $\mathbb{R}^{N \times n}$, but not everywhere on $\mathbb{R}^{N \times n}$ ?

A first answer to this question is contained in the following local regularity result.

Theorem 1.2 (Anzellotti \& Giaquinta [5]). Assume that $f$ is convex with (1.2). Moreover, suppose that $f$ is $C^{2}$ near some point $z_{0}$ in $\mathbb{R}^{N \times n}$ and that $D^{2} f\left(z_{0}\right)$ is positive. Then every minimizer $u \in W^{1, p}\left(\Omega, \mathbb{R}^{N}\right)$ of $F$ is of class $C^{1, \alpha}$ for every $\alpha \in(0,1)$ near every point $x_{0} \in \Omega$ with

$$
f_{B_{r}\left(x_{0}\right)}\left|D u-z_{0}\right|^{p} d x \underset{r \searrow 0}{\longrightarrow} 0 .
$$

Roughly speaking the theorem asserts that for obtaining regularity in those points $x_{0}$ where the Lebesgue value of $D u$ is $z_{0}$ one only needs to impose strict convexity near $z_{0}$. Clearly, since almost-every point is a Lebesgue-point of $D u$ the usual partial regularity (i. e. regularity outside a negligible set if $f$ is strictly convex everywhere on $\mathbb{R}^{N \times n}$ ) is contained as a special case. However, the theorem still contains a global assumption - namely that $f$ be convex — which is not localized near $z_{0}$. Thus, if $f$ fails to be convex, nothing can be deduced. Finally, it should be mentioned that Theorem 1.2 does not only hold for $p>1$ but even covers the limit exponent $p=1$, where $F$ needs to be defined in a generalized way in the space of functions of bounded variation. This is one of the principal points of Theorem 1.2 but we will not discuss it in more detail since it is not relevant for the purposes of the present paper.

Another local regularity result is available for the quasiconvex case:

Theorem 1.3 (Acerbi \& Fusco [3]). Let $p \geq 2$. Assume that $f$ satisfies (1.2) and the Lipschitz condition

$$
\left|f\left(z_{2}\right)-f\left(z_{1}\right)\right| \leq L\left(1+\left|z_{1}\right|+\left|z_{2}\right|\right)^{p-1}\left|z_{2}-z_{1}\right| \quad \text { for } z_{1}, z_{2} \in \mathbb{R}^{N \times n}
$$

with some constant $L$. Moreover, suppose that $f$ is $C^{2}$ near some point $z_{0}$ in $\mathbb{R}^{N \times n}$ and that $f$ is strictly quasiconvex at $z_{0}$. Then every minimizer $u \in W^{1, p}\left(\Omega, \mathbb{R}^{N}\right)$ of $F$ is of class $C^{1, \alpha}$ for every $\alpha \in(0,1)$ near every point $x_{0} \in \Omega$ with

$$
f_{B_{r}\left(x_{0}\right)}\left|D u-z_{0}\right|^{p} d x \underset{r \searrow 0}{\longrightarrow} 0 .
$$


Clearly, the basic idea of localizing the assumptions is visible in both of the two previous theorems. However, also in Theorem 1.3 not all the hypotheses are localized near $z_{0}$ : On the one hand the Lipschitz assumption (1.4) is imposed globally. On the other hand quasiconvexity - and even strict quasiconvexity at a single point - is in its nature a non-local condition, as pointed out in [37]. In particular, the assumptions of Theorem 1.2 do not imply strict quasiconvexity at $z_{0}$ and thus, even if we ignore the restriction $p \geq 2$, Theorem 1.2 is not contained in Theorem 1.3 .

In this paper we replace - in some sense - the finite point $z_{0}$ by $\infty$ and thus deal with localization at infinity. Actually, a growing literature has considered this subject, called asymptotically regular problems: Regularity theory for integrands with a particular structure near infinity has been investigated first in [9] and subsequently in $[30,42,48,26,14,38,10,40,41,47,21,23,24]$. Integrands which become strictly convex near infinity, but without particular structure, have been studied in the scalar case $N=1[20]$ and recently also in the vectorial case $[49,50]$. In particular, a partial regularity result for such integrands - namely that minimizers are Lipschitz continuous on an open and dense subset of $\Omega$ - has been obtained in [50]. We stress that this result is in two aspects optimal: On the one hand no asymptotic regularity result may go beyond Lipschitz continuity ${ }^{1}$. On the other hand a counterexample of [50] shows that it is not possible to establish regularity outside a negligible set (which would be the natural thing to expect); thus, regularity on a dense subset is in some sense optimal.

Here, we consider asymptotically regular problems from a somewhat different point of view and point out the relation to the local regularity results stated above. More precisely, we will exhibit an adequate notion of strict quasiconvexity at infinity which we call asymptotic quasiconvexity. We will establish several characterizations of this notion and we will see that, in contrast to quasiconvexity at a finite point, it is essentially a local condition at $\infty$ (see the discussion after Theorem 2.5). Moreover, we will show that asymptotic quasiconvexity suffices for proving a partial regularity result which generalizes the above mentioned one from [50]. In fact, we will demonstrate that the assumptions of Theorem 1.3 are satisfied in a neighborhood of $\infty$ and thus the asymptotic result can be deduced as a corollary. By the way we utilize the methods of $[8,6,7,16]$ to show that Theorem 1.3 and the asymptotic results extend to the subquadratic case $1<p<2$ which has been excluded in [3, 50]. Additionally, we will discuss the regularity of relaxed minimizers and we will see as a technical side benefit - that the Lipschitz assumption (1.4) can be removed in our setting; see Lemma 2.8 and Theorem 2.11 below.

Moreover, keeping most of the terminology we deal with the case of quasilinear elliptic systems

$$
\operatorname{div} a(D u)=0 \text { on } \Omega,
$$

where the structure function $a: \mathbb{R}^{N \times n} \rightarrow \mathbb{R}^{N \times n}$ is Borel measurable and satisfies the growth condition

$$
|a(z)| \leq L(1+|z|)^{p-1} \quad \text { for } z \in \mathbb{R}^{N \times n} .
$$

Without going into the details let us just mention that many results for weak solutions of such systems are similar and intimately related to analogous developments for minimizers of variational integrals. We focus on the notion of quasimonotonicity which can be defined in formal analogy to the definition of quasiconvexity (see Section 2.3 for a precise statement). Quasimonotonicity is a generalization of the classical notion of monotonicity and related to quasiconvexity by the fact that a

\footnotetext{
${ }^{1}$ To see this one considers an arbitrary Lipschitz continuous function $u$, say with Lipschitz constant $M$, and constructs an asymptotically regular integrand which attains its minimum value everywhere on the whole ball $\overline{B_{M}}$. Then $u$ is obviously a minimizer of the corresponding integral.
} 
function with quasimonotone gradient is quasiconvex. If $a$ is strictly quasimonotone, then the existence and the partial regularity of weak solutions of (1.5) have been proved in [53] and [25,33,34], respectively.

Here, we will establish a local regularity result for non-monotone systems which is the analog of Theorem 1.3 in terms of quasimonotonicity. Furthermore, we will introduce the notion of asymptotic quasimonotonicity and we will show that some of the above-mentioned results can be carried over to asymptotically quasimonotone systems - but sometimes with different proofs.

\section{Definitions and statements}

We recall that the dimensions $n \geq 2, N \geq 1$, the growth exponent $1<\boldsymbol{p}<\infty$, and the bounded open set $\Omega \subset \mathbb{R}^{n}$ are fixed throughout this paper.

\subsection{Quasiconvexity and results for integrals}

Now we specify the notion of quasiconvexity. We restrict ourselves to restating the definitions and refer the reader to $[12,31]$ for further information on quasiconvexity and its applications.

Definition 2.1 (Quasiconvexity). A locally bounded Borel function $f: \mathbb{R}^{N \times n} \rightarrow \mathbb{R}$ is said to be quasiconvex at $z \in \mathbb{R}^{N \times n}$ iff

$$
f_{B_{1}} f(z+D \varphi) d x \geq f(z)
$$

holds for all $\varphi \in C_{\mathrm{cpt}}^{\infty}\left(B_{1}, \mathbb{R}^{N}\right)$. Moreover, $f$ is quasiconvex iff $f$ is quasiconvex at every point in $\mathbb{R}^{N \times n}$.

Next, we give a definition of strict quasiconvexity which is coupled to the growth exponent $p$.

Definition 2.2 (Strict quasiconvexity). Consider a locally bounded Borel function $f: \mathbb{R}^{N \times n} \rightarrow \mathbb{R}$. Then $f$ is said to be strictly quasiconvex at $z \in \mathbb{R}^{N \times n}$ iff there exists a positive constant $\gamma$ such that

$$
f_{B_{1}} f(z+D \varphi) d x \geq f(z)+\gamma f_{B_{1}}(1+|D \varphi|)^{p-2}|D \varphi|^{2} d x
$$

holds for all $\varphi \in C_{\mathrm{cpt}}^{\infty}\left(B_{1}, \mathbb{R}^{N}\right)$. Moreover, $f$ is uniformly strictly quasiconvex iff there exists a positive $\gamma$ such that

$$
f_{B_{1}} f(z+D \varphi) d x \geq f(z)+\gamma f_{B_{1}}(1+|z|+|D \varphi|)^{p-2}|D \varphi|^{2} d x
$$

holds for all $z \in \mathbb{R}^{N \times n}$ and $\varphi \in C_{\mathrm{cpt}}^{\infty}\left(B_{1}, \mathbb{R}^{N}\right)$.

The terminology from the last definition can be understood looking at the model case of the nondegenerate $p$-energy density

$$
e_{p}(z):=\frac{1}{p}\left(1+|z|^{2}\right)^{\frac{p}{2}}
$$

Indeed, $f$ is strictly quasiconvex at $z \in \mathbb{R}^{N \times n}$ iff $f-\delta e_{p}$ is quasiconvex at $z$ for $0<\delta \ll 1$ and $f$ is uniformly strictly quasiconvex iff $f-\delta e_{p}$ is quasiconvex for $0<\delta \ll 1$.

Now we state our results. We start with the extension of Theorem 1.3 to the subquadratic case $1<p<2$ which we will establish in Section 6.2. 
Theorem 2.3 (Local regularity for minimizers). Theorem 1.3 holds verbatim for all $p \in(1, \infty)$.

Next, we introduce the notion of asymptotic quasiconvexity.

Definition 2.4 (Asymptotic quasiconvexity). We say that $f: \mathbb{R}^{N \times n} \rightarrow \mathbb{R}$ is asymptotically quasiconvex iff there exist a positive constant $M$ and a uniformly strictly quasiconvex function $g$ such that

$$
f(z)=g(z) \quad \text { for }|z|>M .
$$

Our first main result is the following characterization of asymptotic quasiconvexity.

Theorem 2.5 (Characterizations of asymptotic quasiconvexity). Under the specified prerequisites each of the following assertions characterizes the asymptotic quasiconvexity of a function $f: \mathbb{R}^{N \times n} \rightarrow \mathbb{R}$ :

If $f$ is $C^{2}$ outside a large ball:

(I) There exists a uniformly strictly quasiconvex function $g$ which is $C^{2}$ outside a large ball with

$$
\lim _{|z| \rightarrow \infty} \frac{\left|D^{2} f(z)-D^{2} g(z)\right|}{|z|^{p-2}}=0 .
$$

If $f$ is locally bounded from below:

(II) There exist a positive constant $M$ and a uniformly strictly quasiconvex function $g$ such that

$$
f(z)=g(z) \quad \text { for }|z|>M
$$

and

$$
g \leq f \quad \text { on } \mathbb{R}^{N \times n}
$$

If $f$ is locally bounded from above:

(III) There exist a positive constant $M$ and a uniformly strictly quasiconvex function $g$ such that

$$
f(z)=g(z) \quad \text { for }|z|>M
$$

and

$$
g \geq f \quad \text { on } \mathbb{R}^{N \times n} .
$$

If $f$ is Borel measurable with growth (1.2):

(IV) There exist positive constants $M, \gamma$, and $L$ such that we have

$$
\begin{aligned}
& \quad f_{B_{1}} f(z+D \varphi) d x \geq f(z)+\gamma f_{B_{1}}(|z|+|D \varphi|)^{p-2}|D \varphi|^{2} d x \quad \text { for }|z|>M \\
& \text { and } \varphi \in C_{\mathrm{cpt}}^{\infty}\left(B_{1}, \mathbb{R}^{N}\right) \text { and } \\
& \quad\left|f\left(z_{2}\right)-f\left(z_{1}\right)\right| \leq L\left(1+\left|z_{1}\right|+\left|z_{2}\right|\right)^{p-1}\left|z_{2}-z_{1}\right| \quad \text { for }\left|z_{1}\right|,\left|z_{2}\right|>M \text {. }
\end{aligned}
$$

We will prove Theorem 2.5 in Section 4.

Asymptotic quasiconvexity itself and the characterization (I) obviously depend only on the values of $f$ near infinity ${ }^{2}$, while the other characterizations seem to

\footnotetext{
${ }^{2} \mathrm{~A}$ more precise formulation is that these properties are preserved if $f$ is changed on a fixed bounded set.
} 
depend, at the first glance, on all the values of $f$. In particular, this is the case for (IV), where $z+D \varphi$ may still be small even if $z$ is large. However, as an interesting outcome of Theorem 2.5 we find that all the characterizations depend only on the values near infinity - at least under the mild boundedness assumptions we imposed in the theorem.

Definition 2.6 (Regular set). For $u \in W^{1, p}\left(\Omega, \mathbb{R}^{N}\right)$ we introduce the regular set

$$
R(u):=\{x \in \Omega: u \text { is Lipschitz near } x\} .
$$

Note that by definition $R(u)$ is open in $\Omega$.

Corollary 2.7 (Partial regularity for minimizers). Assume that $f$ is $C^{2}$ outside a large ball, satisfies the growth condition (1.2) and the Lipschitz condition (1.4), and is asymptotically quasiconvex. Then for every minimizer $u \in W^{1, p}\left(\Omega, \mathbb{R}^{N}\right)$ of $F$ the regular set $R(u)$ is dense in $\Omega$.

Proof. Using characterization (IV) of Theorem 2.5 we find a positive constant $M$ such that the assumptions of Theorem 2.3 are satisfied near every $z_{0} \in \mathbb{R}^{N \times n}$ with $\left|z_{0}\right|>M$. Thus, by Theorem $2.3 u$ is of class $C^{1, \alpha}$ near every point $x_{0} \in \Omega$ with

$$
f_{B_{r}\left(x_{0}\right)}\left|D u-z_{0}\right|^{p} d x \underset{r \searrow 0}{\longrightarrow} 0
$$

for some $z_{0}$ with $\left|z_{0}\right|>M$ and all these points $x_{0}$ belong to $R(u)$.

Now assume for contradiction that some $x \in \Omega$ is not contained in the closure of $R(u)$. Then there is no point $x_{0}$ as before in a whole neighborhood of $x$. Thus, $|D u|$ is essentially bounded by $M$ on this neighborhood and $u$ is Lipschitz near $x$. Consequently, $x \in R(u)$ and we have reached the desired contradiction.

We find it worth remarking that the corollary covers, in particular, integrands which are quasiconvex, but not necessarily strictly quasiconvex on the whole of $\mathbb{R}^{N \times n}$ and thus includes problems with a degenerate ellipticity. For instance, looking at characterization (IV) integrands satisfying the degenerate quasiconvexity condition

$$
f_{B_{1}} f(z+D \varphi) d x \geq f(z)+\gamma f_{B_{1}}(|z|+|D \varphi|-M)_{+}^{p-2}|D \varphi|^{2} d x
$$

for all $z \in \mathbb{R}^{N \times n}$ and $\varphi \in C_{\mathrm{cpt}}^{\infty}\left(B_{1}, \mathbb{R}^{N}\right)$ are included. However, even for such integrands the result that $R(u)$ is dense is still optimal in the aspects discussed above. This follows from the fact that the quasiconvex envelope of the integrand from the counterexample in [50] satisfies (2.2); compare [50, Remark 8.13].

In the light of these applications to degenerate problems Corollary 2.7 should also be compared to the results of $[18,17,51]$ on degenerate quasiconvex problems. In these papers partial $C^{1, \alpha}$-regularity is established for problems with a particular type of degeneration at the origin. In contrast, in Corollary 2.7 we do not impose any particular hypothesis on the structure of the integrand.

However, Corollary 2.7 even holds if the quasiconvexity of $f$ is not only degenerate but does generally fail. In this case one may not hope to prove the existence of minimizers of $F$ (not even if the additional coercivity condition (2.4) below is imposed). Therefore, in the next subsection we will discuss the validity of the result for generalized minimizers. As a byproduct it will also turn out that the hypothesis that $f$ be locally Lipschitz can be weakened. 


\subsection{An extension to generalized minimizers}

For later use we record the following basic regularity property of quasiconvex functions; see [12, Theorem 2.31, Proposition 2.32, Theorem 5.3] and [31, Lemma 5.2].

Lemma 2.8. Quasiconvex functions are locally Lipschitz. Moreover, if $f$ is quasiconvex with growth (1.2) then the Lipschitz condition (1.4) holds, possibly with a larger constant $L$.

Next, following [12, Chapter 9.2.1] we briefly recall the definition of the quasiconvex envelope and the basic idea of relaxation.

Definition 2.9 (Quasiconvex envelope and relaxed minimizer). Assume that $f$ : $\mathbb{R}^{N \times n} \rightarrow \mathbb{R}$ is a locally bounded Borel integrand and that there exists a quasiconvex function $g$ with $g \leq f$ on $\mathbb{R}^{N \times n}$. Then letting

$$
Q f(z):=\sup \left\{h(z): h \text { is a quasiconvex function with } h \leq f \text { on } \mathbb{R}^{N \times n}\right\}
$$

we define another quasiconvex function $Q f: \mathbb{R}^{N \times n} \rightarrow \mathbb{R}$, which is called the quasiconvex envelope of $f$. Moreover, if $f$ and $g$ satisfy the growth conditions ${ }^{3}$

$$
\begin{gathered}
f(z) \leq L(1+|z|)^{p} \\
g(z) \geq-L(1+|z|)^{p}
\end{gathered}
$$

then the relaxed functional

$$
Q F[u]:=\int_{\Omega} Q f(D u) d x
$$

is well-defined for every $u \in W^{1, p}\left(\Omega, \mathbb{R}^{N}\right)$ and we say that $u \in W^{1, p}\left(\Omega, \mathbb{R}^{N}\right)$ is a relaxed minimizer of $F$ iff $u$ is a minimizer of $Q F$.

Now we fix some $u_{0} \in W^{1, p}\left(\Omega, \mathbb{R}^{N}\right)$ and write $\mathscr{D}$ for the Dirichlet class $u_{0}+$ $W_{0}^{1, p}\left(\Omega, \mathbb{R}^{N}\right)$. Then by the relaxation theorem of [11] (see also [1, Section 3] or [12, Theorem 9.1]) we have

$$
\inf _{\mathscr{D}} Q F=\inf _{\mathscr{D}} F \text {. }
$$

Keeping in mind that $Q F \leq F$ by definition we infer that every minimizer is also a relaxed minimizer. Moreover, if the additional coercivity condition

$$
\liminf _{|z| \rightarrow \infty} \frac{f(z)}{|z|^{p}}>0
$$

holds the existence of a relaxed minimizer in $\mathscr{D}$ can be proved by the direct method in the calculus of variations. Thus, in this case the infimum on the left-hand side of (2.3) can be replaced by a minimum and the notion of relaxed minimizers is a reasonable way of defining generalized minimizers of $F$.

After these preparations we are now ready to provide the extension of Corollary 2.7 to generalized minimizers.

Lemma 2.10. Assume that $f: \mathbb{R}^{N \times n} \rightarrow \mathbb{R}$ is Borel measurable, locally bounded and asymptotically quasiconvex. Then $Q f$ is well-defined,

$$
f(z)=Q f(z) \quad \text { for }|z| \gg 1
$$

and $Q f$ is asymptotically quasiconvex.

\footnotetext{
${ }^{3}$ We remark that the lower bound for $g$ is automatically satisfied, possibly with a larger constant $L$; see [36, Lemma 2.5].
} 
Proof. Using characterization (II) of Theorem 2.5 we infer that $Q f$ is well-defined with $f(z)=Q f(z)$ for $|z| \gg 1$. Then asymptotic quasiconvexity of $Q f$ follows easily.

Theorem 2.11 (Partial regularity for relaxed minimizers). Assume that $f: \mathbb{R}^{N \times n} \rightarrow$ $\mathbb{R}$ is $C^{2}$ outside a large ball, Borel measurable with growth (1.2), and asymptotically quasiconvex. Then for every relaxed minimizer $u \in W^{1, p}\left(\Omega, \mathbb{R}^{N}\right)$ of $F$ the regular set $R(u)$ is dense in $\Omega$.

Proof. If $f$ satisfies the assumptions of Theorem 2.11 then by Lemma $2.10 Q f$ is well-defined with growth (1.2) and thus also $Q F$ is well-defined. Furthermore, by Lemma 2.10 and Lemma 2.8 the quasiconvex envelope $Q f$ satisfies all the assumptions of Corollary 2.7. Consequently, Theorem 2.11 follows applying Corollary 2.7 to the relaxed functional $Q F$.

\subsection{Quasimonotonicity and results for systems}

Next we will be concerned with the weak formulation of (1.5). In this case our results are - up to some technical points which will be mentioned below - analogous to the ones for integrals stated in Section 2.1 above. We start specifying the notion of a weak solution and the notion of (strict) quasimonotonicity.

Definition 2.12 (Weak solution). Suppose that a is Borel measurable with growth (1.6). We say that $u \in W^{1, p}\left(\Omega, \mathbb{R}^{N}\right)$ is a weak solution of the system (1.5) iff

$$
\int_{\Omega} a(D u) \cdot D \varphi d x=0
$$

holds for every $\varphi \in W_{0}^{1, p}\left(\Omega, \mathbb{R}^{N}\right)$.

Definition 2.13 (Quasimonotonicity). Consider a locally bounded Borel function $b: \mathbb{R}^{N \times n} \rightarrow \mathbb{R}^{N \times n}$. Then $b$ is said to be quasimonotone at $z \in \mathbb{R}^{N \times n}$ iff

$$
f_{B_{1}} b(z+D \varphi) \cdot D \varphi d x \geq 0
$$

holds for all $\varphi \in C_{\mathrm{cpt}}^{\infty}\left(B_{1}, \mathbb{R}^{N}\right)$. Moreover, $b$ is quasimonotone iff $b$ is quasimonotone at every point in $\mathbb{R}^{N \times n}$.

Definition 2.14 (Strict quasimonotonicity). Consider a locally bounded Borel function $b: \mathbb{R}^{N \times n} \rightarrow \mathbb{R}^{N \times n}$. Then $b$ is said to be strictly quasimonotone at $z \in \mathbb{R}^{N \times n}$ iff there exists a positive constant $\gamma$ such that

$$
f_{B_{1}} b(z+D \varphi) \cdot D \varphi d x \geq \gamma f_{B_{1}}(1+|D \varphi|)^{p-2}|D \varphi|^{2} d x
$$

holds for all $\varphi \in C_{\mathrm{cpt}}^{\infty}\left(B_{1}, \mathbb{R}^{N}\right)$. Moreover, $b$ is strictly quasimonotone near some $z_{0} \in \mathbb{R}^{N \times n}$ if there exists a $\varrho>0$ such that (2.5) holds for all $z \in B_{\varrho}\left(z_{0}\right)$ with a common constant $\gamma>0$. Finally, $b$ is uniformly strictly quasimonotone iff there exists a positive $\gamma$ such that

$$
f_{B_{1}} b(z+D \varphi) \cdot D \varphi d x \geq \gamma f_{B_{1}}(1+|z|+|D \varphi|)^{p-2}|D \varphi|^{2} d x
$$

holds for all $z \in \mathbb{R}^{N \times n}$ and $\varphi \in C_{\mathrm{cpt}}^{\infty}\left(B_{1}, \mathbb{R}^{N}\right)$.

Our local regularity result for systems reads now: 
Theorem 2.15 (Local regularity for weak solutions). Assume that a is Borel measurable and satisfies (1.6). Moreover, suppose that a is $C^{1}$ near some point $z_{0}$ in $\mathbb{R}^{N \times n}$ and strictly quasimonotone near $z_{0}$. Then every weak solution $u \in$ $W^{1, p}\left(\Omega, \mathbb{R}^{N}\right)$ of $(1.5)$ is of class $C^{1, \alpha}$ for every $\alpha \in(0,1)$ near every point $x_{0} \in \Omega$ with

$$
f_{B_{r}\left(x_{0}\right)}\left|D u-z_{0}\right|^{p} d x \underset{r \searrow 0}{\longrightarrow} 0 .
$$

Theorem 2.15 will be proved in Section 6.1.

Clearly, Theorem 2.15 is the analog of Theorem 2.3 for systems. However, the reader should note that in Theorem 2.3 only quasiconvexity at $z_{0}$ was needed while now we imposed quasimonotonicity near - and not only at - $z_{0}$. This difference is basically due to the fact that we did not impose any global continuity condition on $a$ and thus [3, Lemma 2.2] cannot be adapted to the present setting.

Next, we introduce the notion of asymptotic quasimonotonicity.

Definition 2.16 (Asymptotic quasimonotonicity). We say that $a: \mathbb{R}^{N \times n} \rightarrow \mathbb{R}^{N \times n}$ is asymptotically quasimonotone iff there exist a positive constant $M$ and a uniformly strictly quasimonotone function b such that

$$
a(z)=b(z) \quad \text { for }|z|>M .
$$

Theorem 2.17 (The notion of asymptotic quasimonotonicity).

(I) Suppose that a is $C^{1}$ outside a large ball. Then a is asymptotically quasimonotone if and only if there exists a uniformly strictly quasimonotone function $b$ which is $C^{1}$ outside a large ball with

$$
\lim _{|z| \rightarrow \infty} \frac{|D a(z)-D b(z)|}{|z|^{p-2}}=0 .
$$

(II) Assume that a is Borel measurable with growth (1.6). If a is asymptotically quasimonotone then there exist positive constants $M$ and $\gamma$ such that we have

$$
f_{B_{1}} a(z+D \varphi) \cdot D \varphi d x \geq \gamma f_{B_{1}}(|z|+|D \varphi|)^{p-2}|D \varphi|^{2} d x \quad \text { for }|z|>M
$$

and $\varphi \in C_{\mathrm{cpt}}^{\infty}\left(B_{1}, \mathbb{R}^{N}\right)$.

Theorem 2.17 will be proved in Section 5 .

Let us remark that it would be interesting to know whether the condition in Theorem 2.17 (II) can be modified or reformulated in such a way that it provides another characterization of asymptotic quasimonotonicity.

Finally, with the terminology of Definition 2.6 we get:

Corollary 2.18 (Partial regularity for weak solutions). Assume that a is $C^{1}$ outside a large ball, Borel measurable with growth (1.6), and asymptotically quasimonotone. Then for every weak solution $u \in W^{1, p}\left(\Omega, \mathbb{R}^{N}\right)$ of (1.5) the regular set $R(u)$ is dense in $\Omega$.

Proof. Corollary 2.18 follows from Theorem 2.15 and Theorem 2.17 precisely in the same way as Corollary 2.7 was deduced from Theorem 2.3 and Theorem 2.5.

\section{Preliminaries}

Our notation follows essentially the standard conventions and was already used in the previous sections. However, some additional remarks on our terminology are in order: 
We use the letters $c$ and $C$ for positive constants, where usually $c$ is used for small and $C$ for large quantities. The values of these constants are allowed to change from line to line and their dependencies will only occasionally be highlighted.

Moreover, for $x_{0} \in \mathbb{R}^{n}$ and $r>0$ we write $B_{r}\left(x_{0}\right)$ for the open ball $\left\{x \in \mathbb{R}^{n}\right.$ : $\left.\left|x-x_{0}\right|<r\right\}$ in $\mathbb{R}^{n}$ with center $x_{0}$ and radius $r$. Similarly, for $z_{0} \in \mathbb{R}^{N \times n}$ we use $B_{r}\left(z_{0}\right)$ for the ball $\left\{z \in \mathbb{R}^{N \times n}:\left|z-z_{0}\right|<r\right\}$ in the space $\mathbb{R}^{N \times n}$ of $(N \times n)$ matrices, where $|\cdot|$ denotes the Euclidean norm on this space. If the center of a ball is 0 we omit it and write just $B_{r}$.

Furthermore, we use the usual symbols $L^{p}$ for Lebesgue spaces, $W^{1, p}$ for firstorder Sobolev spaces, $C^{k}$ for the space of functions with continuous derivatives up to order $k$, and $C^{1, \alpha}$ for first-order Hölder spaces. The arguments following these symbols indicate the domain of definition and the target space of the functions under consideration and the subscripts $\mathrm{cpt}$ and ${ }_{0}$ refer to the subspaces of functions with compact support and zero boundary values, respectively.

We will extensively use the abbreviations

$$
V_{z}(\xi):=(1+|z|+|\xi|)^{\frac{p-2}{2}} \xi \quad \text { and } \quad V(\xi):=V_{0}(\xi)=(1+|\xi|)^{\frac{p-2}{2}} \xi .
$$

We note that

$$
\left|V_{z}(t)\right|^{2} \text { is non-decreasing in } t \geq 0
$$

and that for $p \geq 2$ the quantity

$$
\left|V_{z}(\xi)\right|^{2}
$$

can be estimated from above and below (with constants depending only on $p$ ) by

$$
(1+|z|)^{p-2}|\xi|^{2}+|\xi|^{p}
$$

The following lemma adapts some arguments from [26] and [50, Lemma 5.1].

Lemma 3.1. Let $M>0$. There is a constant $K_{M}>M$, depending only on $M$ and $p$, such that for $z \in \mathbb{R}^{N \times n}, \varphi \in W^{1, p}\left(B_{1}, \mathbb{R}^{N}\right)$ and $K \geq K_{M}$ we have

$$
|z|>K \Longrightarrow|\{|z+D \varphi| \leq M\}| \leq \frac{1}{K} \int_{B_{1}}\left|V_{z}(D \varphi)\right|^{2} d x .
$$

Proof. We choose $K_{M}>M$ large enough that for all $K \geq K_{M}$ we have

$$
\left|V_{K}(K-M)\right|^{2} \geq K \text {. }
$$

Then for $|z|>K$ and $y \in B_{1}$ with $|z+D \varphi(y)| \leq M$ there holds

$$
K-M \leq|D \varphi(y)|
$$

and using (3.1) we arrive at

$$
1 \leq \frac{1}{K}\left|V_{z}(K-M)\right|^{2} \leq \frac{1}{K}\left|V_{z}(D \varphi(y))\right|^{2} .
$$

Now the claim follows by integration.

The next lemma is a restatement of [4, Lemma 2.1].

Lemma 3.2. For $1<p<2$ and $z, \xi \in \mathbb{R}^{N \times n}$ there holds

$$
\int_{0}^{1}(1+|z+t \xi|)^{p-2} d t \leq C(1+|z|+|\xi|)^{p-2}
$$

with some positive constant $C$ depending only on $p$. 
Lemma 3.3. For $1<p<2$ and $z, \xi \in \mathbb{R}^{N \times n}$ there holds

$$
\int_{0}^{1} \int_{0}^{1}(1+|z+s t \xi|)^{p-2} d s t d t \leq \bar{C}(1+|z|+|\xi|)^{p-2}
$$

with some positive constant $\bar{C}$ depending only on $p$.

Proof. Using Lemma 3.2 twice we find

$$
\begin{aligned}
\int_{0}^{1} \int_{0}^{1}(1+|z+s t \xi|)^{p-2} d s t d t & \leq C \int_{0}^{1}(1+|z|+|t \xi|)^{p-2} t d t \\
& \leq C \int_{0}^{1}(1+|z+t \xi|)^{p-2} d t \leq C^{2}(1+|z|+|\xi|)^{p-2}
\end{aligned}
$$

and the claim is proved.

Moreover, we need versions of Young's inequality and the triangle inequality for $V$; for $p \geq 2$ the proofs are straightforward and for $p<2$ they can be found for instance in [17].

Lemma 3.4. For all $z, \xi \in \mathbb{R}^{N \times n}$ there hold

$$
\begin{gathered}
(1+|\xi|)^{p-2}|\xi||z| \leq C\left[|V(\xi)|^{2}+|V(z)|^{2}\right] \\
|V(\xi+z)|^{2} \leq C\left[|V(\xi)|^{2}+|V(z)|^{2}\right]
\end{gathered}
$$

with a constant $C$ depending only $p$.

The following lemma is a variant of [2, Lemma II.3]

Lemma 3.5. Assume that $a: \mathbb{R}^{N \times n} \rightarrow \mathbb{R}^{N \times n}$ is $C^{1}$ on $B_{2 \varrho}\left(z_{0}\right)$ and fulfills (1.6). Then one has

$$
|a(\xi)-a(z)| \leq C(1+|\xi|)^{p-2}|\xi-z| \quad \text { for all } z \in B_{\varrho}\left(z_{0}\right) \text { and } \xi \in \mathbb{R}^{N \times n},
$$

where $C$ depends on $p, L, \varrho,\left|z_{0}\right|$, and $\sup _{B_{3 \varrho / 2}\left(z_{0}\right)}|D a|$.

Proof. We distinguish the cases $|\xi-z| \leq \frac{1}{2} \varrho$ and $|\xi-z|>\frac{1}{2} \varrho$. In the first case, we clearly have

$$
|a(\xi)-a(z)| \leq|\xi-z| \sup _{B_{3 \varrho / 2}\left(z_{0}\right)}|D a| \leq C\left(\varrho,\left|z_{0}\right|\right)(1+|\xi|)^{p-2}|\xi-z| \sup _{B_{3 \varrho / 2}\left(z_{0}\right)}|D a|,
$$

while in the second case there hold

$$
\frac{1+|\xi|}{|\xi-z|} \leq \frac{1+|z|+|\xi-z|}{|\xi-z|}=\frac{1+|z|}{|\xi-z|}+1 \leq C\left(\varrho,\left|z_{0}\right|\right)
$$

and

$$
\begin{aligned}
|a(\xi)-a(z)| & \leq L(1+|\xi|)^{p-1}+L(1+|z|)^{p-1} \\
& \leq C\left(p, L, \varrho,\left|z_{0}\right|\right)(1+|\xi|)^{p-1} \leq C\left(p, L, \varrho,\left|z_{0}\right|\right)(1+|\xi|)^{p-2}|\xi-z| .
\end{aligned}
$$

We will rely on the following decay estimate which corresponds to a regularity result for linear comparison systems. 
Lemma 3.6 (An estimate for linear elliptic systems). Suppose that $a: \mathbb{R}^{N \times n} \rightarrow$ $\mathbb{R}^{N \times n}$ is strictly quasimonotone at $A \in \mathbb{R}^{N \times n}$ with constant $\gamma>0$ and $C^{1}$ near $A$. Then for $v \in W^{1,1}\left(B_{1}, \mathbb{R}^{N}\right)$ with

$$
\int_{B_{1}} D a(A) D v \cdot D \varphi d x=0 \quad \text { for all } \varphi \in C_{\mathrm{cpt}}^{\infty}\left(B_{1}, \mathbb{R}^{N}\right)
$$

there holds

$$
f_{B_{2 \tau}}\left|D v-(D v)_{2 \tau}\right|^{2} d x \leq C \tau^{2}\left(f_{B_{1}}|D v| d x\right)^{2} \quad \text { for all } 0<\tau<\frac{1}{4},
$$

where $C$ depends only on $n, N, \gamma$ and an upper bound for $|D a(A)|$.

Proof. The strict quasimonotonicity of $a$ at $A$ can be reformulated saying that for each $\varphi \in C_{\mathrm{cpt}}^{\infty}\left(B_{1}, \mathbb{R}^{N}\right)$ the expression

$$
\Gamma(t):=f_{B_{1}} a(A+t D \varphi) \cdot t D \varphi d x-\gamma f_{B_{1}}(1+|t D \varphi|)^{p-2} t^{2}|D \varphi|^{2} d x,
$$

defined for $t \in \mathbb{R}$, attains a minimum for $t=0$. We deduce

$$
0 \leq \Gamma^{\prime \prime}(0)=f_{B_{1}} D a(A) D \varphi \cdot D \varphi d x-2 \gamma f_{B_{1}}|D \varphi|^{2} d x,
$$

that is the quadratic function $\mathbb{R}^{N \times n} \rightarrow \mathbb{R}, z \mapsto D a(A) z \cdot z-2|z|^{2}$ is quasiconvex on $\mathbb{R}^{N \times n}$. By [12, Theorem 5.3] it follows that this function is also rank-1-convex which means that the Legendre-Hadamard ellipticity condition

$$
D a(A) z \cdot z \geq 2 \gamma|z|^{2} \quad \text { holds for all rank-1-matrices } z \in \mathbb{R}^{N \times n} \text {. }
$$

Consequently, we may combine a classical regularity result (see [31, Section 10.2]) with [6, Proposition 2.10] to get

$$
\begin{aligned}
f_{B_{2 \tau}}\left|D v-(D v)_{2 \tau}\right|^{2} d x & \leq C \tau^{2} f_{B_{1 / 2}}\left|D v-(D v)_{\frac{1}{2}}\right|^{2} d x \\
& \leq C \tau^{2} \sup _{B_{1 / 2}}|D v|^{2} \leq C \tau^{2}\left(f_{B_{1}}|D v| d x\right)^{2} .
\end{aligned}
$$

Next we come to a Sobolev-Poincaré inequality for the subquadratic case which is formulated in terms of the function $V$. Extending earlier considerations in [8] such an inequality has been established in [6, Theorem 2.4] for all $1<p<2$, but with different balls on the left-hand and right-hand side. The following refined version involves just one ball and is taken from [16, Theorem 2].

Lemma 3.7 (Sobolev-Poincaré type inequality). Let $1<p<2$. Setting $p^{\#}:=$ $\frac{2 n}{n-p}>2$ for all $u \in W^{1, p}\left(B_{r}, \mathbb{R}^{N}\right)$ there holds

$$
\left(f_{B_{r}}\left|V\left(\frac{u-u_{r}}{r}\right)\right|^{p^{\#}}\right)^{\frac{1}{p \#}} d x \leq C\left(f_{B_{r}}|V(D u)|^{2} d x\right)^{\frac{1}{2}} .
$$

Here, the positive constant $C$ depends only on $n, N$ and $p$.

Finally, we restate [6, Lemma 2.7]: 
Lemma 3.8 (Iteration lemma). Consider $v \in L^{p}\left(B_{2 r} ; \mathbb{R}^{N}\right)$ and a bounded function $g:[r, 2 r] \rightarrow[0, \infty)$ and assume that for some $K \geq 0, \vartheta<1$ and all $r \leq s<t<2 r$ one has

$$
g(s) \leq \vartheta g(t)+K \int_{B_{2 r}}\left|V\left(\frac{v}{t-s}\right)\right|^{2} d x .
$$

Then the following estimate holds true:

$$
g(r) \leq C K \int_{B_{2 r}}\left|V\left(\frac{v}{r}\right)\right|^{2} d x .
$$

Here, $C$ denotes a positive constant depending only on $p$ and $\vartheta$.

\section{Asymptotic quasiconvexity}

In this section we establish the characterizations of asymptotic quasiconvexity stated in Theorem 2.5.

Proof of Theorem 2.5. We start proving the theorem in the case $p \geq 2$. The changes which are needed in the case $p<2$ will be outlined at the end of the proof.

If $f$ is $C^{2}$ outside a large ball then obviously asymptotic quasiconvexity implies (I). We shall now prove that conversely (I) implies asymptotic quasiconvexity. Let $g$ be as in (I), in particular uniformly strictly quasiconvex with some constant $\gamma>0$. By scaling we may assume that $f$ and $g$ are $C^{2}$ outside $\overline{B_{1 / 2}}$. Now we set

$$
h:=f-g
$$

and note that $h$ is $C^{2}$ outside $\overline{B_{1 / 2}}$ with

$$
\lim _{|z| \rightarrow \infty} \frac{D^{2} h(z)}{|z|^{p-2}}=0 .
$$

We claim that there hold

$$
\lim _{|z| \rightarrow \infty} \frac{D h(z)}{|z|^{p-1}}=0
$$

and

$$
\lim _{|z| \rightarrow \infty} \frac{h(z)}{|z|^{p}}=0 .
$$

To verify (4.2) we abbreviate $\bar{z}:=\frac{z}{|z|}$ and estimate

$$
\begin{aligned}
& \frac{|D h(z)|}{|z|^{p-1}} \leq \frac{1}{|z|^{p-1}}\left[\int_{0}^{1}\left|D^{2} h(\bar{z}+t(z-\bar{z}))\right| d t|z-\bar{z}|+|D h(\bar{z})|\right] \\
& \quad \leq \int_{0}^{1} \frac{\left|D^{2} h(\bar{z}+t(z-\bar{z}))\right|}{|\bar{z}+t(z-\bar{z})|^{p-2}} d t+\frac{|D h(\bar{z})|}{|z|^{p-1}} \\
& \quad \leq \int_{0}^{\frac{1}{\sqrt{|z|}}} \frac{\left|D^{2} h(\bar{z}+t(z-\bar{z}))\right|}{|\bar{z}+t(z-\bar{z})|^{p-2}} d t+\int_{\frac{1}{\sqrt{|z|}}}^{1} \frac{\left|D^{2} h(\bar{z}+t(z-\bar{z}))\right|}{|\bar{z}+t(z-\bar{z})|^{p-2}} d t+\frac{|D h(\bar{z})|}{|z|^{p-1}} \\
& \quad \leq \frac{1}{\sqrt{|z|}} \sup _{|y|>1} \frac{\left|D^{2} h(y)\right|}{|y|^{p-2}}+\sup _{|y|>\sqrt{|z|}} \frac{\left|D^{2} h(y)\right|}{|y|^{p-2}}+\frac{\max _{|y|=1}|D h(y)|}{|z|^{p-1}} \underset{|z| \rightarrow \infty}{\longrightarrow} 0 .
\end{aligned}
$$

Here, we used (4.1) in the last step to conclude both $\sup _{|y|>1} \frac{\left|D^{2} h(y)\right|}{|y|^{p-2}}<\infty$ and $\sup _{|y|>\sqrt{|z|}} \frac{\left|D^{2} h(y)\right|}{|y|^{p^{-2}}} \rightarrow 0$. Reasoning analogously, one obtains (4.3) from (4.2). Now 
we fix a smooth cut-off function $\eta: \mathbb{R}^{N \times n} \rightarrow \mathbb{R}$ with $\mathbb{1}_{\mathbb{R}^{N \times n} \backslash B_{2}} \leq \eta \leq \mathbb{1}_{\mathbb{R}^{N \times n} \backslash B_{1}}$ on $\mathbb{R}^{N \times n}$ and set $K:=\max \left\{\sup _{\mathbb{R}^{N \times n}}|D \eta|, \sup _{\mathbb{R}^{N \times n}}\left|D^{2} \eta\right|\right\}$. Then letting $\eta_{M}(z):=$ $\eta(z / M)$ for $M>0$ we have

$$
\left|D \eta_{M}\right| \leq \frac{K}{M} \quad \text { and } \quad\left|D^{2} \eta_{M}\right| \leq \frac{K}{M^{2}} \quad \text { on } \mathbb{R}^{N \times n} .
$$

Using (4.1), (4.2), and (4.3) we choose $M>1$ large enough such that for $|z| \geq M$ we have

$$
\begin{aligned}
\left|D^{2} h(z)\right| & \leq \frac{\gamma}{3}|z|^{p-2} \\
|D h(z)| & \leq \frac{\gamma}{6 K}|z|^{p-1}, \\
|h(z)| & \leq \frac{\gamma}{6 K}|z|^{p} .
\end{aligned}
$$

Hence, we get for $M \leq|z| \leq 2 M$

$\left|D^{2}\left(\eta_{M} h\right)(z)\right| \leq \eta_{M}(z)\left|D^{2} h(z)\right|+2\left|D \eta_{M}(z)\right||D h(z)|+\left|D^{2} \eta_{M}(z)\right||h(z)| \leq \frac{5 \gamma}{3}|z|^{p-2}$.

Next, we observe that $\left|D^{2}\left(\eta_{M} h\right)(z)\right| \leq \frac{5 \gamma}{3}|z|^{p-2}$ holds for all $z \in \mathbb{R}^{N \times n}$ since it holds trivially for $|z|<M$ or $|z|>M$. Writing

$$
\begin{aligned}
\left(\eta_{M} h\right)(z+D \varphi)-\left(\eta_{M} h\right)(z)-D & \left(\eta_{M} h\right)(z) D \varphi \\
& =\int_{0}^{1} \int_{0}^{1} D^{2}\left(\eta_{M} h\right)(z+s t D \varphi) d s t d t(D \varphi, D \varphi)
\end{aligned}
$$

we find that this implies in particular

$$
\begin{aligned}
f_{B_{1}}\left(\eta_{M} h\right)(z+D \varphi) d x & \geq\left(\eta_{M} h\right)(z)-\frac{5 \gamma}{3} f_{B_{1}} \int_{0}^{1} \int_{0}^{1}|z+s t D \varphi|^{p-2} d s t d t|D \varphi|^{2} d x \\
& \geq\left(\eta_{M} h\right)(z)-\frac{5 \gamma}{6} f_{B_{1}}\left|V_{z}(D \varphi)\right|^{2} d x
\end{aligned}
$$

for all $z \in \mathbb{R}^{N \times n}$ and $\varphi \in C_{\mathrm{cpt}}^{\infty}\left(B_{1}, \mathbb{R}^{N}\right)$. Consequently, $\tilde{g}:=g+\eta_{M} h$ is still strictly quasiconvex with $\tilde{g}(z)=f(z)$ for $|z|>2 M$. This proves the asymptotic quasiconvexity of $f$ and establishes the characterization (I).

Next, we deal with the characterization (II). Here it suffices to prove that asymptotic quasiconvexity of $f$ implies (II) since the converse implication holds trivially. Thus we assume that there exist a constant $M$ and a uniformly strictly quasiconvex $g$ with $f(z)=g(z)$ for $|z|>M$. To fix notations, we assume that (2.1) holds for $g$ with a constant $\gamma>0$, and we let $K:=\sup _{|z| \leq M}(g-f)(z)$; note that $K<\infty$ since $f$ is locally bounded from below and $g$ is locally bounded. Now we denote by $h$ a smooth non-negative function on $\mathbb{R}^{N \times n}$ with compact support such that we have $\left|D^{2} h\right| \leq \gamma$ on $\mathbb{R}^{N \times n}$ and $h(z) \geq K$ for $|z| \leq M$. Then arguing essentially as for (4.4) we find that $\tilde{g}:=g-h$ is uniformly strictly quasiconvex with $\tilde{g}=f$ near infinity and $\tilde{g} \leq f$ on $\mathbb{R}^{N \times n}$. Thus, (II) holds.

The arguments used for (II) apply analogously for the characterization (III).

Finally, we assume that $f$ is Borel measurable and locally bounded and establish the characterization in (IV). Using Lemma 2.8 it is easily seen that (II) implies (IV) and it suffices to show that a function $f$ with (IV) is asymptotically quasiconvex. To this aim we suppose that $f$ satisfies (IV) and we first introduce

$$
\tilde{f}(z):=f(z)-\varepsilon\left(1+|z|^{2}\right)^{\frac{p}{2}} \quad \text { for } z \in \mathbb{R}^{N \times n} .
$$


Here, $\varepsilon>0$ is chosen sufficiently small (depending only on $n, N, p$, and $\gamma$ ) that both conditions in (IV) still hold for $\tilde{f}$, say with constants $M, \frac{\gamma}{2}$, and $L+\gamma$. Next we let

$$
\tilde{g}(z):=\inf \left\{f_{B_{1}} \tilde{f}(z+D \varphi) d x: \varphi \in C_{\mathrm{cpt}}^{\infty}\left(B_{1}, \mathbb{R}^{N}\right)\right\} \quad \text { for } z \in \mathbb{R}^{N \times n} .
$$

We note that this specification defines a function $\tilde{g}: \mathbb{R}^{N \times n} \rightarrow \mathbb{R} \cup\{-\infty\}$ with $\tilde{g} \leq \tilde{f}$ on $\mathbb{R}^{N \times n}$ and $\tilde{g}(z)=\tilde{f}(z)$ for $|z|>M$. In order to show that $\tilde{g}$ is locally bounded from below we fix some $z \in \mathbb{R}^{N \times n}$ with $|z| \leq M$ and choose an arbitrary $\tilde{z} \in \mathbb{R}^{N \times n}$ with $|\tilde{z}|=2 M$. Then using both conditions in (IV) and local boundedness for $\tilde{f}$ we find

$$
\begin{aligned}
& f_{B_{1}} \tilde{f}(z+D \varphi) d x \\
& \geq f_{B_{1}} \tilde{f}(\tilde{z}+D \varphi) d x+\frac{1}{\left|B_{1}\right|} \int_{\{|D \varphi|>3 M\}}[\tilde{f}(z+D \varphi)-\tilde{f}(\tilde{z}+D \varphi)] d x-C \\
& \geq \tilde{f}(\tilde{z})+\frac{\gamma}{4} f_{B_{1}}|D \varphi|^{p} d x-C f_{B_{1}}|D \varphi|^{p-1} d x-C \\
& \geq-C .
\end{aligned}
$$

Here, we also employed Young's inequality in the last step, and $C$ denotes a positive constant which depends only on $p, \gamma, L, M$, and $\varepsilon$ but whose precise value may change from occurrence to occurrence. Anyway, (4.5) shows $\tilde{g}(z) \geq-C$ for $|z| \leq$ $M$. Recalling $\tilde{g}(z)=\tilde{f}(z) \geq-(L+\varepsilon)(1+|z|)^{p}$ for $|z|>M$ this proves local boundedness of $\tilde{g}$ from below. Having obtained this boundedness it follows by Dacorogna's formula ${ }^{4}$ that $\tilde{g}$ coincides with the quasiconvex envelope $Q \tilde{f}$ of $\tilde{f}$ and is thus quasiconvex. At this stage it is not difficult to verify that

$$
g(z):=\tilde{g}(z)+\varepsilon\left(1+|z|^{2}\right)^{\frac{p}{2}} \quad \text { for } z \in \mathbb{R}^{N \times n}
$$

defines a uniformly strictly quasiconvex function $g$ with $g(z)=f(z)$ for $|z|>M$. We have thus established the asymptotic quasiconvexity of $f$ and the characterization (IV).

Finally, we point out how to modify the above arguments in the subquadratic case $p<2$. The first point above where a change is needed is inequality (4.4). In fact, revisiting the arguments preceding (4.4) we find a constant $M$ such that we have $\left|D^{2}\left(\eta_{M} h\right)(z)\right| \leq \frac{\gamma}{2 \bar{C}}(1+|z|)^{p-2}$ for all $z \in \mathbb{R}^{N \times n}$, where $\bar{C}$ denotes the constant from Lemma 3.3. Using this together with Lemma 3.3 in the derivation of (4.4) we arrive at

$$
f_{B_{1}}\left(\eta_{M} h\right)(z+D \varphi) d x \geq\left(\eta_{M} h\right)(z)-\frac{\gamma}{2} f_{B_{1}}\left|V_{z}(D \varphi)\right|^{2} d x .
$$

The remainder of the proof of the characterization (I) can now be carried over to the case $p<2$. In the arguments used for (II) we need to replace the requirement $\left|D^{2} h\right| \leq \gamma$ on $\mathbb{R}^{N \times n}$ with $\left|D^{2} h(z)\right| \leq \frac{\gamma}{2 \bar{C}}(1+|z|)^{p-2}$ for all $z \in \mathbb{R}^{N \times n}$, where $\bar{C}$ is again the constant from Lemma 3.3. However, a function $h$ with the required properties can still be constructed easily. Moreover, we said above that the uniform strict

\footnotetext{
${ }^{4}$ Adaptable versions of Dacorogna's formula are [11, Theorem 6.9] and [35, Proposition 8.1]. Unfortunately, both statements do not immediately apply to our situation (where $\tilde{g}$ is merely locally bounded from below), but one may argue as follows: In [11, Theorem 6.9] it is assumed that there exists a quasiconvex function below $\tilde{f}$, and the verification of this hypothesis is not immediate in our situation. However, we may still apply the theorem since the missing hypothesis is only needed to conclude that $\tilde{g}$ is locally bounded from below (and thus it is itself such a function). Furthermore, [35, Proposition 8.1] is formulated only for continuous functions $\tilde{f}$, but it is not difficult to adapt the proof to our setting.
} 
quasiconvexity of $\tilde{g}:=g-h$ can be verified following the arguments around (4.4). This is still true for $p<2$, but requires - as before - an additional application of Lemma 3.3. Finally, in the proof of the characterization (IV) Lemma 3.3 is needed once more and (4.5) deserves an additional remark in the case $p<2$ : Actually, the estimate holds as stated, but to see that the second inequality is true one should note that for $p<2$ the term arising from the strict quasiconvexity can be handled using the elementary inequality $(2 M+|D \varphi|)^{p-2}|D \varphi|^{2} \geq \frac{1}{2}|D \varphi|^{p}-(2 M)^{p}$.

\section{$5 \quad$ Asymptotic quasimonotonicity}

In this section we derive Theorem 2.17.

Proof of Theorem 2.17. The proof of the characterization (I) is essentially the same as the proof of characterization (I) in Theorem 2.5. The reader should note that in contrast to Theorem 2.5 we now need to deal only with first, but not with second derivatives. Thus the required calculations are essentially simplified versions of the ones already presented and we will skip the details. Let us just mention that to cope with the subquadratic setting it suffices to use Lemma 3.2 instead of Lemma 3.3 .

However, for (II) it is impossible to follow the arguments used for characterization (IV) of Theorem 2.5 which exploited the fact that the integrands are real-valued. Instead we shall provide a different proof: We assume that $a$ is asymptotically quasimonotone, that is $a$ coincides with a function $b$ outside $\overline{B_{M}}$, where $b$ is uniformly strictly quasimonotone with constant $\gamma$. Then for $z \in \mathbb{R}^{N \times n}$ and $\varphi \in C_{\mathrm{cpt}}^{\infty}\left(B_{1}, \mathbb{R}^{N}\right)$ we have

$$
\begin{aligned}
f_{B_{1}} a(z+D \varphi) \cdot D \varphi d x & =f_{B_{1}} b(z+D \varphi) \cdot D \varphi d x+R \\
& \geq \gamma f_{B_{1}}\left|V_{z}(D \varphi)\right|^{2} d x+R,
\end{aligned}
$$

where we introduced the abbreviation

$$
R:=\frac{1}{\left|B_{1}\right|} \int_{\{|z+D \varphi| \leq M\}}[a(z+D \varphi)-b(z+D \varphi)] \cdot D \varphi d x .
$$

To estimate $R$ we assume $|z|>K \geq \max \left\{2 M+1, K_{M}\right\}$, where $K_{M}$ is the constant from Lemma 3.1 and $K$ will be fixed later. We note that $|z+D \varphi| \leq M$ can only hold where $|D \varphi| \geq \max \{|z|-M, 1\} \geq \frac{1}{2}(|z|+1)$ is satisfied and get

$$
\begin{aligned}
R & \geq-\frac{2 L(1+M)^{p-1}}{\left|B_{1}\right|} \int_{\{|z+D \varphi| \leq M\}}|D \varphi| d x \\
& \geq-\frac{2 L(1+M)^{p-1}}{\left|B_{1}\right|} C \int_{\{|z+D \varphi| \leq M\}}\left|V_{z}(D \varphi)\right|^{\frac{2}{p}} d x \\
& \geq-2 L(1+M)^{p-1} C\left(\frac{|\{|z+D \varphi| \leq M\}|}{\left|B_{1}\right|}\right)^{\frac{p-1}{p}}\left(f_{B_{1}}\left|V_{z}(D \varphi)\right|^{2} d x\right)^{\frac{1}{p}} \\
& \geq-2 L\left(\frac{1+M}{K^{\frac{1}{p}}}\right)^{p-1} C f_{B_{1}}\left|V_{z}(D \varphi)\right|^{2} d x
\end{aligned}
$$

where we used Hölder's inequality and Lemma 3.1 in the last steps. Here the constant $C$ depends only on $p$ (and can be chosen as 1 for $p \geq 2$ ). Consequently, if we choose $K$ large enough, depending only on $p, L$, and $M$, then we have

$$
R \geq-\frac{\gamma}{2} f_{B_{1}}\left|V_{z}(D \varphi)\right|^{2} d x
$$


and

$$
f_{B_{1}} a(z+D \varphi) \cdot D \varphi d x \geq \frac{\gamma}{2} f_{B_{1}}\left|V_{z}(D \varphi)\right|^{2} d x
$$

for $|z|>K$. Now we combine this with the elementary inequality

$$
\left|V_{z}(D \varphi)\right|^{2} \geq c(|z|+|D \varphi|)^{p-2}|D \varphi|^{2}
$$

for $|z|>K$, where $c$ depends only on $p$ and $K$ (again $c=1$ works for $p \geq 2$ ), and arrive at the claim of (II).

\section{Proofs of the local regularity results}

In this section we prove Theorem 2.15, the local regularity result for systems, and we discuss how the arguments need to be modified to establish the variational result in Theorem 2.3. We follow the approach of [3] and involve ideas of $[8,6,16]$ to handle the subquadratic case $p<2$.

\subsection{Proof of Theorem 2.15}

Throughout this subsection we will assume that $u \in W^{1, p}\left(\Omega, \mathbb{R}^{N}\right)$ is a given weak solution of (1.5), where $a$ satisfies the assumptions of Theorem 2.15. In particular, fixing $z_{0} \in \mathbb{R}^{N \times n}$, and $x_{0} \in \Omega$, we suppose that (2.6) holds for $x_{0}$ and $z_{0}$ and assume that $a$ is $C^{1}$ near $z_{0}$ - say on $B_{2 \varrho}\left(z_{0}\right)$ - and strictly quasimonotone near $z_{0}$ - say at every point in $B_{2 \varrho}\left(z_{0}\right)$ with a common constant $\gamma$ as in Definition 2.14.

Lemma 6.1 (Caccioppoli inequality). For $A \in B_{\varrho}\left(z_{0}\right)$ and $B_{2 r}\left(y_{0}\right) \subset \Omega$ there holds

$$
f_{B_{r}\left(y_{0}\right)}|V(D u-A)|^{2} d x \leq C f_{B_{2 r}\left(y_{0}\right)}\left|V\left(\frac{u(x)-\zeta-A\left(x-y_{0}\right)}{r}\right)\right|^{2} d x
$$

for all $\zeta \in \mathbb{R}^{N}$. Here $C=C\left(p, L, \gamma, \varrho,\left|z_{0}\right|, \sup _{B_{3 \varrho / 2}\left(z_{0}\right)}|D a|\right)$ is a positive constant.

Proof. We assume $y_{0}=0$ and consider radii $r \leq s<t<2 r$. We let

$$
\varphi(x)=(u(x)-\zeta-A x) \eta(x),
$$

where $\eta$ is a smooth cut-off function with $\mathbb{1}_{B_{s}} \leq \eta \leq \mathbb{1}_{B_{t}}$ and $|\nabla \eta| \leq \frac{2}{t-s}$. We note $\varphi \in W_{0}^{1, p}\left(B_{t}, \mathbb{R}^{N}\right)$ and $A+D \varphi=D u$ on $B_{s}$. Using this together with the quasimonotonicity $^{5}$ of $a$ and the fact that $u$ is a solution of (1.5) we find

$$
\begin{aligned}
\gamma \int_{B_{t}}|V(D \varphi)|^{2} d x \leq & \int_{B_{t}}[a(A+D \varphi)-a(D u)] \cdot D \varphi d x \\
= & \int_{B_{t} \backslash B_{s}}[a(A+D \varphi)-a(D u)] \cdot D \varphi d x \\
\leq & \int_{B_{t} \backslash B_{s}}|a(A+D \varphi)-a(A)||D \varphi| d x \\
& \quad+\int_{B_{t} \backslash B_{s}}|a(D u)-a(A)||D \varphi| d x
\end{aligned}
$$

\footnotetext{
${ }^{5}$ The reader should note that by scaling and translation the unit ball in the definition of quasimonotonicity may be replaced by an arbitrary ball in $\mathbb{R}^{n}$. Moreover, since (1.6) is assumed a simple approximation argument allows the use of $W_{0}^{1, p}$-test functions $\varphi$ in the quasimonotonicity condition.
} 
Now we note $|A| \leq C\left(\left|z_{0}\right|, \varrho\right)$ and

$$
|D \varphi(x)| \leq|D u(x)-A|+2 \frac{|u(x)-\zeta-A x|}{t-s} .
$$

Then we apply Lemma 3.5 and the estimates from Lemma 3.4 to the right-hand side of (6.1). We come out with

$$
\begin{aligned}
& \gamma \int_{B_{s}}|V(D u-A)|^{2} d x \\
\leq & C\left[\int_{B_{t} \backslash B_{s}}(1+|A+D \varphi|)^{p-2}|D \varphi|^{2} d x+\int_{B_{t} \backslash B_{s}}(1+|D u|)^{p-2}|D u-A||D \varphi| d x\right] \\
\leq & C\left[\int_{B_{t} \backslash B_{s}}|V(D \varphi)|^{2} d x+\int_{B_{t} \backslash B_{s}}(1+|D u-A|)^{p-2}|D u-A||D \varphi| d x\right] \\
\leq & C\left[\int_{B_{t} \backslash B_{s}}|V(D \varphi)|^{2} d x+\int_{B_{t} \backslash B_{s}}|V(D u-A)|^{2} d x\right] \\
\leq & C\left[\int_{B_{t}}\left|V\left(\frac{u(x)-\zeta-A x}{t-s}\right)\right|^{2} d x+\int_{B_{t} \backslash B_{s}}|V(D u-A)|^{2} d x\right]
\end{aligned}
$$

We fill the hole by adding to both sides of (6.2) the quantity $C \int_{B_{s}}|V(D u-A)|^{2} d x$ in order to get

$$
\int_{B_{s}}|V(D u-A)|^{2} d x \leq \int_{B_{t}}\left|V\left(\frac{u(x)-\zeta-A x}{t-s}\right)\right|^{2} d x+\vartheta \int_{B_{t}}|V(D u-A)|^{2} d x,
$$

where $\vartheta=\frac{C}{1+C}$ holds for the the constant $C$ from the last line of (6.2). Since $\vartheta$ is smaller than 1 we conclude by an application of Lemma 3.8 that

$$
\int_{B_{r}}|V(D u-A)|^{2} d x \leq C \int_{B_{2 r}}\left|V\left(\frac{u-\zeta-A x}{r}\right)\right|^{2} d x
$$

holds.

Now we define the excess function as

$$
E(x, r)=f_{B_{r}(x)}\left|V\left(D u-(D u)_{x, r}\right)\right|^{2} d x .
$$

Proposition 6.2 (Decay estimate). There exists a positive constant $C_{0}$ such that for every $0<\tau<\frac{1}{4}$ there exists a $\kappa \in(0,1)$ with the following property. Whenever for a ball $B_{r}\left(x_{0}\right) \subset \Omega$ there hold

$$
\left|(D u)_{x_{0}, r}-z_{0}\right|+\left|(D u)_{x_{0}, \tau r}-z_{0}\right|<\varrho \quad \text { and } \quad E\left(x_{0}, r\right) \leq \kappa
$$

then we have

$$
E\left(x_{0}, \tau r\right) \leq C_{0} \tau^{2} E\left(x_{0}, r\right) .
$$

Here, the dependency of $C_{0}$ is given by $C_{0}\left(n, N, p, L, \gamma, \varrho,\left|z_{0}\right| \sup _{B_{3 e / 2}\left(z_{0}\right)}|D a|\right)$.

Proof. We argue by contradiction and assume that for some $C_{0}$ to be chosen later the proposition is false. Then there exist $0<\tau<\frac{1}{4}$ and a sequence of balls $B_{r_{j}}\left(x_{j}\right) \subset \Omega$ such that

$$
\left|(D u)_{x_{j}, r_{j}}-z_{0}\right|+\left|(D u)_{x_{j}, \tau r_{j}}-z_{0}\right|<\varrho \quad \text { and } \quad \lambda_{j}^{2}:=E\left(x_{j}, r_{j}\right) \underset{j \rightarrow \infty}{\longrightarrow} 0
$$


but

$$
E\left(x_{j}, \tau r_{j}\right)>C_{0} \tau^{2} \lambda_{j}^{2}
$$

In particular, the reader should note that we have $\lambda_{j}>0$ since otherwise $u$ would be affine on $B_{r_{j}}\left(x_{j}\right)$ and (6.5) would not hold. Moreover, we may and will assume $\lambda_{j} \leq 1$ for all $j \in \mathbb{N}$.

\section{Step 1. Blow up.}

Setting $A_{j}:=(D u)_{x_{j}, r_{j}}, \zeta_{j}:=(u)_{x_{j}, r_{j}}$ and

$$
v_{j}(y):=\frac{u\left(x_{j}+r_{j} y\right)-\zeta_{j}-r_{j} A_{j} y}{\lambda_{j} r_{j}}
$$

for all $y \in B_{1}$, one can easily check that $\left|A_{j}-z_{0}\right|<\varrho,\left(D v_{j}\right)_{1}=0$ and $\left(v_{j}\right)_{1}=0$. In particular $\left|A_{j}\right|$ is bounded by a constant which depends only on $\left|z_{0}\right|$ and $\varrho$. By the definition of $\lambda_{j}$ in (6.4), we get

$$
f_{B_{1}}\left(1+\lambda_{j}\left|D v_{j}\right|\right)^{p-2}\left|D v_{j}\right|^{2} d x=1
$$

Introducing the abbreviation

$$
p_{2}:=\min \{2, p\}
$$

it follows that we have

$$
f_{B_{1}}\left|D v_{j}\right|^{p_{2}} d x \leq C(p)
$$

and

$$
f_{B_{1}} \lambda_{j}^{p}\left|D v_{j}\right|^{p} d x \underset{j \rightarrow \infty}{\longrightarrow} 0 .
$$

Therefore, passing if necessary to subsequences - which we do not relabel for simplicity of notation - we deduce

$$
\begin{aligned}
A_{j} \rightarrow A & \text { in } \mathbb{R}^{N n} \\
v_{j} \rightarrow v & \text { weakly in } W^{1, p_{2}}\left(B_{1}, \mathbb{R}^{N}\right) \text { and strongly in } L^{p_{2}}\left(B_{1}, \mathbb{R}^{N}\right) \\
\lambda_{j}\left|D v_{j}\right| \rightarrow 0 & \text { a. e. on } B_{1},
\end{aligned}
$$

where the limit objects $A$ and $v$ satisfy $|A-z| \leq \varrho$ and

$$
\left(f_{B_{1}}|D v| d x\right)^{p_{2}} \leq f_{B_{1}}|D v|^{p_{2}} d x \leq C(p) .
$$

Finally, using (6.6) again we may assume in the case $p>2$ that $\lambda_{j}^{\frac{p-2}{p}} v_{j}$ converges strongly in $L^{p}\left(B_{1} \cdot \mathbb{R}^{N}\right)$ as $j \rightarrow \infty$. Taking into account $\lambda_{j} \underset{j \rightarrow \infty}{\longrightarrow} 0$ and $v_{j} \underset{j \rightarrow \infty}{\longrightarrow} v$ strongly in $L^{2}\left(B_{1}, \mathbb{R}^{N}\right)$ this implies

$$
\lambda_{j}^{p-2} \int_{B_{1}}\left|v_{j}\right|^{p} d x \underset{j \rightarrow \infty}{\longrightarrow} 0 .
$$

Step 2. $v$ solves a linear system.

Using that $u$ solves the system (1.5) we have

$$
\begin{aligned}
0 & =\frac{1}{\lambda_{j}^{2} r_{j}^{n}} \int_{B_{r_{j}}\left(x_{j}\right)}\left[a(D u)-a\left(A_{j}\right)\right] \cdot D \varphi_{j} d x \\
& =\frac{1}{\lambda_{j}} \int_{B_{1}}\left[a\left(A_{j}+\lambda_{j} D v_{j}\right)-a\left(A_{j}\right)\right] \cdot D \varphi d x
\end{aligned}
$$


where $\varphi \in C_{\mathrm{cpt}}^{\infty}\left(B_{1}, \mathbb{R}^{N}\right)$ is arbitrary and $\varphi_{j}(x):=\lambda_{j} r_{j} \varphi\left(\frac{x-x_{j}}{r_{j}}\right)$. Next we split

$$
B_{1}=E_{j}^{+} \cup E_{j}^{-},
$$

where

$$
\begin{aligned}
& E_{j}^{+}:=\left\{y \in B_{1}: \lambda_{j}\left|D v_{j}(y)\right|>\frac{1}{2} \varrho\right\}, \\
& E_{j}^{-}:=\left\{y \in B_{1}: \lambda_{j}\left|D v_{j}(y)\right| \leq \frac{1}{2} \varrho\right\} .
\end{aligned}
$$

We note that (6.7) gives

$$
\left|E_{j}^{+}\right| \leq\left(\frac{2}{\varrho}\right)^{p_{2}} \lambda_{j}^{p_{2}} \int_{E_{j}^{+}}\left|D v_{j}\right|^{p_{2}} d x \leq C(p, \varrho) \lambda_{j}^{p_{2}} \underset{j \rightarrow \infty}{\longrightarrow} 0
$$

and observe

$$
\begin{aligned}
0= & \frac{1}{\lambda_{j}} \int_{E_{j}^{+}}\left[a\left(A_{j}+\lambda_{j} D v_{j}\right)-a\left(A_{j}\right)\right] \cdot D \varphi d x \\
& \quad+\frac{1}{\lambda_{j}} \int_{E_{j}^{-}}\left[a\left(A_{j}+\lambda_{j} D v_{j}\right)-a\left(A_{j}\right)\right] \cdot D \varphi d x \\
= & I_{j}+I I_{j}
\end{aligned}
$$

By Lemma 3.5 and the elementary inequality

$$
\frac{1}{1+\left|A_{j}\right|}\left(1+\lambda_{j}\left|D v_{j}\right|\right) \leq 1+\left|A_{j}+\lambda_{j} D v_{j}\right| \leq\left(1+\left|A_{j}\right|\right)\left(1+\lambda_{j}\left|D v_{j}\right|\right)
$$

we have

$$
\begin{aligned}
\left|I_{j}\right| & \leq C\|D \varphi\|_{\infty} \int_{E_{j}^{+}}\left(1+\left|A_{j}+\lambda_{j} D v_{j}\right|\right)^{p-2}\left|D v_{j}\right| d x \\
& \leq C\|D \varphi\|_{\infty} \int_{E_{j}^{+}}\left(1+\lambda_{j}\left|D v_{j}\right|\right)^{p-2}\left|D v_{j}\right| d x \\
& \leq C\|D \varphi\|_{\infty} \lambda_{j} \int_{E_{j}^{+}}\left(1+\lambda_{j}\left|D v_{j}\right|\right)^{p-2}\left|D v_{j}\right|^{2} d x \\
& \leq C\|D \varphi\|_{\infty} \lambda_{j} \underset{j \rightarrow \infty}{\longrightarrow} 0,
\end{aligned}
$$

while for $I I_{j}$ we find by (6.7) and the dominated convergence theorem

$$
\begin{aligned}
&\left|I I_{j}-\int_{E_{j}^{-}} D a(A) D v_{j} \cdot D \varphi d x\right| \\
&=\left|\int_{E_{j}^{-}} \int_{0}^{1}\left[D a\left(A_{j}+t \lambda_{j} D v_{j}\right)-D a(A)\right] d t D v_{j} \cdot D \varphi d x\right| \\
& \leq\left(\int_{E_{j}^{-}} \int_{0}^{1}\left|D a\left(A_{j}+t \lambda_{j} D v_{j}\right)-D a(A)\right|^{\frac{p_{2}}{p_{2}-1}} d t d x\right)^{\frac{p_{2}-1}{p_{2}}}\left(\int_{B_{1}}\left|D v_{j}\right|^{p_{2}}\right)^{\frac{1}{p_{2}}}\|D \varphi\|_{\infty} \\
& \underset{j \rightarrow \infty}{\longrightarrow} 0 .
\end{aligned}
$$

We conclude

$$
I I_{j} \underset{j \rightarrow \infty}{\longrightarrow} \int_{B_{1}} D a(A) D v \cdot D \varphi d x
$$

and combining (6.10), (6.11) and (6.12) we find

$$
0=\int_{B_{1}} D a(A) D v \cdot D \varphi d x
$$


that is $v$ weakly solves a linear system of partial differential equations with constant coefficients. By Lemma 3.6 and (6.8) we get

$$
f_{B_{2 \tau}}\left|D v-(D v)_{2 \tau}\right|^{2} d x \leq C \tau^{2},
$$

where the constant $C$ in this decay estimate depends only on $n, N, \gamma$ and an upper bound for $\operatorname{Da}(A)$.

\section{Step 3. Conclusion.}

We apply the Caccioppoli inequality of Lemma 6.1 with $\zeta_{j}+r_{j} \lambda_{j}\left(v_{j}\right)_{2 \tau}$ instead of $\zeta$ and $(D u)_{x_{j}, \tau r_{j}}$ instead of $A$ (recall $\left|(D u)_{x_{j}, \tau r_{j}}-z_{0}\right|<\varrho$ ) in order to have

$$
\begin{aligned}
\frac{E\left(x_{j}, \tau r_{j}\right)}{\lambda_{j}^{2}} & =\frac{1}{\lambda_{j}^{2}} f_{B_{\tau r_{j}\left(x_{j}\right)}}\left|V\left(D u-(D u)_{x_{j}, \tau r_{j}}\right)\right|^{2} d x \\
& \leq \frac{C}{\lambda_{j}^{2}} f_{B_{2 \tau r_{j}\left(x_{j}\right)}}\left|V\left(\frac{u(x)-\zeta_{j}-r_{j} \lambda_{j}\left(v_{j}\right)_{2 \tau}-(D u)_{x_{j}, \tau r_{j}}\left(x-x_{j}\right)}{\tau r_{j}}\right)\right|^{2} d x
\end{aligned}
$$

Noting that $(D u)_{x_{j}, \tau r_{j}}=A_{j}+\lambda_{j}\left(D v_{j}\right)_{\tau}$ and changing variable, we obtain

$$
\limsup _{j \rightarrow \infty} \frac{E\left(x_{j}, \tau r_{j}\right)}{\lambda_{j}^{2}} \leq \limsup _{j \rightarrow \infty} \frac{C}{\lambda_{j}^{2}} f_{B_{2 \tau}}\left|V\left(\frac{\lambda_{j}\left(v_{j}(y)-\left(v_{j}\right)_{2 \tau}-\left(D v_{j}\right)_{\tau} y\right)}{\tau}\right)\right|^{2} d y .
$$

In the case $p>2$ we now use $v_{j} \rightarrow v$ strongly in $L^{2}\left(B_{1}, \mathbb{R}^{N}\right)$ and (6.9) and deduce

$$
\begin{aligned}
\limsup _{j \rightarrow \infty} \frac{E\left(x_{j}, \tau r_{j}\right)}{\lambda_{j}^{2}} \leq \limsup _{j \rightarrow \infty} C & {\left[f_{B_{2 \tau}}\left|\frac{v_{j}(y)-\left(v_{j}\right)_{2 \tau}-\left(D v_{j}\right)_{\tau} y}{\tau}\right|^{2} d y\right.} \\
& \left.+\lambda_{j}^{p-2} f_{B_{2 \tau}}\left|\frac{v_{j}(y)-\left(v_{j}\right)_{2 \tau}-\left(D v_{j}\right)_{\tau} y}{\tau}\right|^{p} d y\right] \\
= & \frac{C}{\tau^{2}} f_{B_{2 \tau}}\left|v-(v)_{2 \tau}-(D v)_{\tau} y\right|^{2} d y .
\end{aligned}
$$

In the case $p \leq 2$ we argue in a different way and use an idea of [6]. We first estimate by the interpolation inequality, Lemma 3.7 , and (6.6)

$$
\begin{aligned}
& \frac{1}{\lambda_{j}^{2}} \int_{B_{1}}\left|V\left(\lambda_{j}\left(v_{j}-v\right)\right)\right|^{2} d y \\
& \quad \leq\left(\frac{1}{\lambda_{j}} \int_{B_{1}}\left|V\left(\lambda_{j}\left(v_{j}-v\right)\right)\right| d y\right)^{2 t}\left(\frac{1}{\lambda_{j}^{p \#}} \int_{B_{1}}\left|V\left(\lambda_{j}\left(v_{j}-v\right)\right)\right|^{p^{\#}} d y\right)^{\frac{2(1-t)}{p^{\#}}} \\
& \quad \leq C\left(\int_{B_{1}}\left|v_{j}-v\right| d y\right)^{2 t}\left(\frac{1}{\lambda_{j}^{2}} \int_{B_{1}}\left|V\left(\lambda_{j}\left(D v_{j}-D v\right)\right)\right|^{2} d y\right)^{1-t} \\
& \underset{j \rightarrow \infty}{\longrightarrow} 0 .
\end{aligned}
$$

Using the triangle inequality from Lemma 3.4 and this convergence in (6.14) we infer

$$
\begin{aligned}
\limsup _{j \rightarrow \infty} \frac{E\left(x_{j}, \tau r_{j}\right)}{\lambda_{j}^{2}} & \leq \limsup _{j \rightarrow \infty} \frac{C}{\lambda_{j}^{2}} f_{B_{2 \tau}}\left|V\left(\frac{\lambda_{j}\left(v(y)-\left(v_{j}\right)_{2 \tau}-\left(D v_{j}\right)_{\tau} y\right)}{\tau}\right)\right|^{2} d y \\
& \leq \frac{C}{\tau^{2}} f_{B_{2 \tau}}\left|v(y)-(v)_{2 \tau}-(D v)_{\tau} y\right|^{2} d y .
\end{aligned}
$$


Now we come back to the general case, where $p$ is arbitrary in $(1, \infty)$, and we note that in conclusion we have proved

$$
\limsup _{j \rightarrow \infty} \frac{E\left(x_{j}, \tau r_{j}\right)}{\lambda_{j}^{2}} \leq \frac{C}{\tau^{2}} f_{B_{2 \tau}}\left|v(y)-(v)_{2 \tau}-(D v)_{\tau} y\right|^{2} d y .
$$

for all these values of $p$. Now, a simple use of Poincaré's inequality yields

$$
\begin{aligned}
\lim _{j \rightarrow \infty} \frac{E\left(x_{j}, \tau r_{j}\right)}{\lambda_{j}^{2}} \leq & \frac{C}{\tau^{2}} f_{B_{2 \tau}}\left|v(y)-(v)_{2 \tau}-(D v)_{2 \tau} y\right|^{2} d y \\
& +\frac{C}{\tau^{2}} f_{B_{2 \tau}}\left|(D v)_{2 \tau} y-(D v)_{\tau} y\right|^{2} d y \\
\leq & C f_{B_{2 \tau}}\left|D v-(D v)_{2 \tau}\right|^{2} d y \\
\leq & C \tau^{2}
\end{aligned}
$$

where we applied (6.13) in the last line. Choosing $C_{0}$ larger than $C$ in the last line and recalling (6.5) we have reached a contradiction. This ends the proof of the proposition.

Once Proposition 6.2 is established the remainder of the proof of Theorem 2.15 consists essentially of standard arguments and follows the lines of [3, pp. 614 \& 615]. While in the case $p \geq 2$ the arguments from [3] apply verbatim, three minor changes are needed to handle the case $p<2$ (still using the excess from (6.3)): The first change concerns the estimate in the proof of [3, Proposition 2.7] which controls the distance of the mean values from the regular point. Here, to get the function $V$ and the excess involved we additionally use the elementary inequality

$$
|z| \leq C(p)\left[|V(z)|+|V(z)|^{\frac{2}{p}}\right]
$$

applied with $D u-(D u)_{\tau^{k} r}$ in place of $z$. The second change is similar in its nature and occurs when [3, Proposition 2.7] is applied in the concluding part of the proof. Indeed, we use (6.16) as before to verify that the hypothesis of the proposition are verified. Finally, the third change regards the deduction of the Hölder continuity at the very end of the proof and follows quite closely [6, p. 163]. Factually, we apply - once more - (6.16) and Hölder's inequality to convert the final decay estimate for the excess into an estimate for the simpler quantity $f_{B_{r}(x)}\left|D u-(D u)_{x, r}\right| d x$. The Hölder continuity of $D u$ then follows from the latter estimate.

\subsection{On the proof of Theorem 2.3}

A proof of Theorem 2.3 can be given along the lines of [3], adapting the ideas of $[8,6]$ for the treatment of the subquadratic setting $1<p<2$. Indeed, the reasoning is quite close to the proof of Theorem 2.15 which we provided above - for the full range $1<p<\infty$. Thus, instead of working out all the details we just point out the necessary modifications in [3, Section 2]:

All the arguments need to be rewritten in terms of the function $V$. In particular, the function $g_{p}$ in [3] is to be replaced by $|V|^{2}$. Let us explain in some detail the changes needed in the individual lemmas:

Changing the meaning of $g_{p}$ as just mentioned, both the statement and the proof of [3, Lemma 2.2] extend without difficulty to the case $1<p<2$. The same assertion is true for [3, Lemma 2.3] (compare [6, Lemma 3.3]), where now $|\eta|^{2}+\lambda^{p-2}|\eta|^{p}$ is to be substituted by $\lambda^{-2}|V(\lambda \eta)|^{2}$ and $|\eta|+\lambda^{p-2}|\eta|^{p-1}$ by $(1+\lambda|\eta|)^{p-2}|\eta|$. Moreover, [3, Lemma 2.4] can be replaced by Lemma 3.8 from our preliminaries section. 
Continuing with such modifications, also [3, Lemma 2.5] can be adapted to the case $1<p<2$. Here, the reader should note that for our purposes only the case $\nu=0$ is relevant and thus both the lemma and its proof are essentially analogous to Lemma 6.1 which we used in the case of systems. Next, [3, Proposition 2.6] can be extended where the only major change occurs in the passage to the limit. Here, the formulas $[3,(2.13)-(2.15)]$ need to be replaced by the interpolation argument (6.15) based on Lemma 3.7 which we already used in the proof of Proposition 6.2. Finally, the necessary modifications in [3, Proposition 2.7] and the conclusion of the proof have already been described at the end of the previous subsection.

\section{Acknowledgements}

This research project was started when T. S. visited Naples in March 2009. He thanks N. FusCo and A. PASSARELli Di NAPOLI for the invitation and gratefully acknowledges the kind hospitality experienced at "Dipartimento di Matematica e Applicazioni R. Caccioppoli". This visit and the research of A. V. were partially supported by the European Research Council under Advanced Grant 226234 "Analytic Techniques for Geometric and Functional Inequalities". Last but not least the authors are indebted to G. Mingione for drawing their attention to the paper [3] in a very early stage of the project.

\section{References}

[1] E. Acerbi, N. Fusco: Semicontinuity problems in the calculus of variations. Arch. Ration. Mech. Anal. 86, 125-145 (1984).

[2] E. ACERBI, N. FusCo: A regularity theorem for minimizers of quasiconvex integrals. Arch. Ration. Mech. Anal. 99, 261-281 (1987).

[3] E. Acerbi, N. Fusco: Local regularity for minimizers of non convex integrals. Ann. Sc. Norm. Super. Pisa, Cl. Sci., IV. Ser. 16, 603-636 (1989).

[4] E. AcerBi, N. Fusco: Regularity for minimizers of nonquadratic functionals: the case $1<p<2$. J. Math. Anal. Appl. 140, 115-135 (1989).

[5] G. Anzellotti, M. Giaquinta: Convex functionals and partial regularity. Arch. Ration. Mech. Anal. 102, 243-272 (1988).

[6] M. Carozza, N. Fusco, G. Mingione: Partial regularity of minimizers of quasiconvex integrals with subquadratic growth. Ann. Mat. Pura Appl., IV. Ser. 175, 141-164 (1998).

[7] M. Carozza, G. Mingione: Partial regularity of minimizers of quasiconvex integrals with subquadratic growth: the general case. Ann. Pol. Math. 77, 219-243 (2001).

[8] M. Carozza, A. Passarelli di Napoli: A regularity theorem for quasiconvex integrals: the case $1<p<2$. Proc. R. Soc. Edinb., Sect. A 126, 1181-1199 (1996).

[9] M. Снipot, L.C. Evans: Linearization at infinity and Lipschitz estimates for certain problems in the calculus of variations. Proc. R. Soc. Edinb., Sect. A 102, 291-303 (1986).

[10] G. Cupini, M. Guidorzi, E. Mascolo: Regularity of minimizers of vectorial integrals with $p-q$ growth. Nonlinear Anal., Theory Methods Appl. 54, 591-616 (2003).

[11] B. Dacorogna: Quasiconvexity and relaxation of nonconvex problems in the calculus of variations. J. Funct. Anal. 46, 102-118 (1982). 
[12] B. Dacorogna: Direct Methods in the Calculus of Variations, Second Edition. Springer, New York, 2008.

[13] E. De Giongi: Un esempio di estremali discontinue per un problema variazionale di tipo ellittico. Boll. Unione Mat. Ital., IV. Ser. 1, 135-137 (1968).

[14] G. Dolzmann, J. Kristensen: Higher integrability of minimizing Young measures. Calc. Var. Partial Differential Equations 22, 283-301 (2005).

[15] F. Duzana, A. Gastel, J.F. Grotowski: Partial regularity for almost minimizers of quasi-convex integrals. SIAM J. Math. Anal. 32, 665-687 (2000).

[16] F. Duzana, J.F. Grotowski, M. Kronz: Regularity of almost minimizers of quasi-convex variational integrals with subquadratic growth. Ann. Mat. Pura Appl., IV. Ser. 184, 421-448 (2005).

[17] F. Duzane, G. Mingione: Regularity for degenerate elliptic problems via p-harmonic approximation. Ann. Inst. Henri Poincaré, Anal. Non Linéaire 21, 735-766 (2004).

[18] L. Esposito, G. Mingione: Partial regularity for minimizers of degenerate polyconvex energies. J. Convex Anal. 8, 1-38 (2001).

[19] L.C. Evans: Quasiconvexity and partial regularity in the calculus of variations. Arch. Ration. Mech. Anal. 95, 227-252 (1986).

[20] I. Fonseca, N. Fusco, P. Marcellini: An existence result for a nonconvex variational problem via regularity. ESAIM, Control Optim. Calc. Var. 7, 69-95 (2002).

[21] M. Foss: Global regularity for almost minimizers of nonconvex variational problems. Ann. Mat. Pura Appl., IV. Ser. 187, 263-321 (2008).

[22] M. Foss, G. Mingione: Partial continuity for elliptic problems. Ann. Inst. Henri Poincaré, Anal. Non Linéaire 25, 471-503 (2008).

[23] M. Foss, A. Passarelli di Napoli, A. Verde: Global Morrey regularity results for asymptotically convex variational problems. Forum Math. 20, 5, 921-953 (2008).

[24] M. Foss, A. Passarelli di Napoli, A. Verde: Global Lipschitz regularity for almost minimizers of asymptotically convex variational problems. Ann. Mat. Pura Appl., IV. Ser. 189, 127-162 (2010).

[25] M. FuCHS: Regularity theorems for nonlinear systems of partial differential equations under natural ellipticity conditions. Analysis 7, 83-93 (1987).

[26] M. Fuchs: Lipschitz regularity for certain problems from relaxation. Asymptotic Analysis 12, 145-151 (1996).

[27] N. Fusco, J.E. Hutchinson: $C^{1, \alpha}$ partial regularity of functions minimising quasiconvex integrals. Manuscr. Math. 54, 121-143 (1986).

[28] N. Fusco, J.E. Hutchinson: Partial regularity in problems motivated by nonlinear elasticity. SIAM J. Math. Anal. 22, 1516-1551 (1991).

[29] M. Giaquinta, G. Modica: Partial regularity of minimizers of quasiconvex integrals. Ann. Inst. Henri Poincaré, Anal. Non Linéaire 3, 185-208 (1986).

[30] M. Giaquinta, G. Modica: Remarks on the regularity of the minimizers of certain degenerate functionals. Manuscr. Math. 57, 55-99 (1986).

[31] E. Giusti: Direct Methods in the Calculus of Variations. World Scientific Publishing Co., New York, 2003.

[32] E. Giusti, M. Miranda: Sulla regolarita delle soluzioni deboli di una classe di sistemi ellittici quasi-lineari. Arch. Ration. Mech. Anal. 31, 173-184 (1968). 
[33] C. Hamburger: Quasimonotonicity, regularity and duality for nonlinear systems of partial differential equations. Ann. Mat. Pura Appl., IV. Ser. 169, 321-354 (1995).

[34] C. Hamburger: Partial regularity of solutions of nonlinear quasimonotone systems. Hokkaido Math. J. 32, 291-316 (2003).

[35] D. Kinderlehrer, P. Pedregal: Characterizations of Young measures generated by gradients. Arch. Ration. Mech. Anal. 115, 329-365 (1991).

[36] J. KRISTEnsen: Lower semicontinuity in spaces of weakly differentiable functions. Math. Ann. 313, 653-710 (1999).

[37] J. Kristensen: On the non-locality of quasiconvexity. Ann. Inst. Henri Poincaré, Anal. Non Linéaire 16, 1-13 (1999).

[38] J. Kristensen, A. TAheri: Partial regularity of strong local minimizers in the multi-dimensional calculus of variations. Arch. Ration. Mech. Anal. 170, 1, 63-89 (2003).

[39] J. Kristensen, G. Mingione: The singular set of minima of integral functionals. Arch. Ration. Mech. Anal. 180, 331-398 (2006).

[40] J. Kristensen, G. Mingione: The singular set of Lipschitzian minima of multiple integrals. Arch. Ration. Mech. Anal. 184, 341-369 (2007).

[41] C. Leone, A. Passarelli di NApoli, A. Verde: Lipschitz regularity for some asymptotically subquadratic problems. Nonlinear Anal. 67, 1532-1539 (2007).

[42] R. Malek-Madani, P.D. Smith: Lipschitz continuity of local minimizers of a nonconvex functional. Appl. Anal. 28, 223-230 (1988).

[43] C.B. Morrey: Quasi-convexity and the lower semicontinuity of multiple integrals. Pac. J. Math. 2, 25-53 (1952).

[44] C.B. Morrey: Partial regularity results for non-linear elliptic systems. J. Math. Mech. 17, 649-670 (1968).

[45] S. Müller, V. SverÁK: Convex integration for Lipschitz mappings and counterexamples to regularity. Ann. Math. (2) 157,715-742 (2003).

[46] J. NEČAs Example of an irregular solution to a nonlinear elliptic system with analytic coefficients and conditions for regularity. Theor. Nonlin. Oper., Constr. Aspects, Proc. int. Summer Sch., Berlin 1975, 197-206 (1977).

[47] A. Passarelli di Napoli, A. Verde: A regularity result for asymptotically convex problems with lower order terms. J. Convex Anal. 15, 131-148 (2008).

[48] J.-P. RAYMOND: Lipschitz regularity of solutions of some asymptotically convex problems. Proc. R. Soc. Edinb., Sect. A 117, 59-73 (1991).

[49] C. Scheven, T. Schmidt: Asymptotically regular problems I: Higher integrability. J. Differ. Equations, 248, 745-791 (2010).

[50] C. Scheven, T. Schmidt: Asymptotically regular problems II: Partial Lipschitz continuity and a singular set of positive measure. Ann. Sc. Norm. Super. Pisa, Cl. Sci. (5) VIII, 469-507 (2009).

[51] T. SchmidT: Regularity theorems for degenerate quasiconvex energies with $(p, q)$-growth. Adv. Calc. Var. 1, 241-270 (2008).

[52] V. Sverák, X. Yan: Non-Lipschitz minimizers of smooth uniformly convex functionals. Proc. Natl. Acad. Sci. USA 99, 15269-15276 (2002).

[53] K. ZHANG: On the Dirichlet problem for a class of quasilinear elliptic systems of partial differential equations in divergence form. Partial differential equations, Proc. Symp., Tianjin/China, 1986, Lect. Notes Math. 1306, 262-277 (1988). 